\title{
GLOMERULAR DISEASE
}

\section{Antiproteinuric efficacy of $A$. manihot superior to losartan}

New data from the first randomized controlled trial of the traditional Chinese medicine Abelmoschus manihot suggest that this herb is more effective than the angiotensin-receptor blocker losartan in reducing proteinuria in patients with primary glomerular disease. A. manihot has previously been reported to ameliorate interstitial renal fibrosis, proteinuria and haematuria, inhibit immune reactions and reduce inflammatory injury in patients with various kidney diseases.

"Use of traditional Chinese medicines to treat chronic kidney disease (CKD) has a long history and $A$. manihot flowers were included in the Compendium of Materia Medica more than four centuries ago," says researcher Xiangmei Chen. "As traditional medicines are generally preferred by Chinese patients, conducting high-quality randomized controlled trials of these therapies to obtain efficacy and safety data is very important."

In their multicentre, open-label trial, Chen and colleagues randomly assigned
417 patients with biopsy-proven primary glomerular disease, stages 1-2 CKD and non-nephrotic range proteinuria to receive either A.manihot (2.5 g huangkui capsule three times per day), losartan potassium (50 mg per day) or both of these therapies. After 24 weeks of treatment, they found significantly greater reductions in 24-h proteinuria from baseline in the A. manihot and combined therapy groups versus the losartan monotherapy group ( $P=0.003$ and $P<0.001$, respectively). Subgroup analysis in patients with $\operatorname{IgA}$ nephropathy (51.5\% of the cohort) gave similar results. No significant changes in mean estimated glomerular filtration rate from baseline and no severe adverse events were reported in any group.

The researchers are now conducting a clinical trial of $A$. manihot in patients with stages 1-4 CKD and are investigating the renoprotective mechanisms of flavinoid monomers - the main biologically active components of A. manihot-in vitro and in animal models. They also plan to test

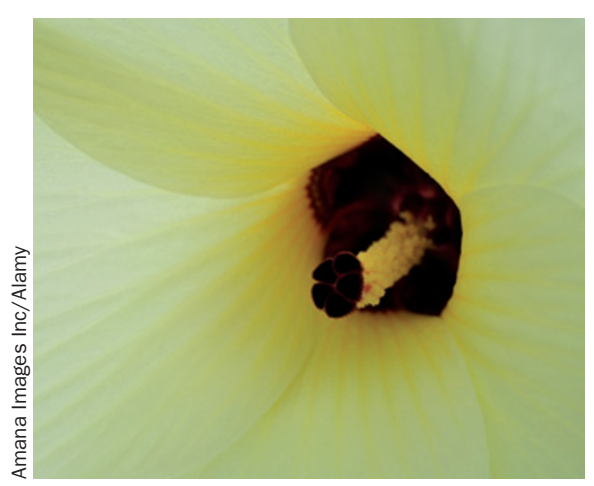

the efficacy of $A$. manihot in patients with secondary kidney diseases, including diabetic nephropathy. "Standardized traditional Chinese medicines such as A. manihot may have a bright future in the treatment of CKD," concludes Chen. Ellen F. Carney

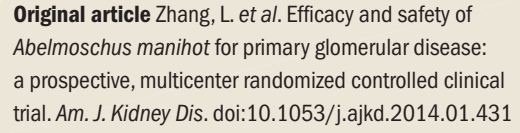

